\title{
Aspirin for the primary prevention of cardiovascular diseases
}

\author{
Ludovica Tagliabue $\cdot$ Franca Dipaola $\cdot$ \\ Francesca Perego • Gian Marco Podda • \\ Gruppo di Autoformazione Metodologica (GrAM)
}

Received: 23 March 2012/ Accepted: 5 May 2012/Published online: 6 June 2012

(C) SIMI 2012

\section{Background}

For secondary prevention of fatal and non-fatal cardiovascular events, benefits of aspirin (ASA) are well defined, and form the basis for current clinical practice [1-4]. In patients without a history of cardiovascular diseases (CVD), in contrast, the picture is not clear: several metaanalyses $[5,6]$ and the individual data meta-analysis of the antithrombotic trialists' (ATT) collaboration [7] show that the efficacy of ASA in reducing the risks of acute myocardial infarction (MI) and ischemic stroke does not outweigh the associated increased risk of bleeding. The conflicting recommendations from guidelines panels reflect this uncertainty $[3,8,9]$. After the publication of ATT study in 2009 [7], three more randomized controlled studies (RCT) evaluating the use of ASA in primary prevention were published [10-12]. Recently, three nearly contemporaneous meta-analyses [13-15] combined old and newest trials' results.

\footnotetext{
L. Tagliabue

Scuola di Specializzazione in Igiene e Medicina Preventiva, Università degli Studi di Milano, Milan, Italy

e-mail: ludovica.tagliabue@gmail.com

F. Dipaola

Medicina Interna, "Istituti Clinici di Perfezionamento",

Sesto S. Giovanni, Milan, Italy

F. Perego

Medicina III, Ospedale "Luigi Sacco",

Università degli Studi di Milano, Milan, Italy

G. M. Podda $(\square)$

Medicina 3, Ospedale San Paolo,

Università degli Studi di Milano, Milan, Italy

e-mail: gmpodda@gmail.com
}

\section{Summary}

We discuss three meta-analyses published between January 2011 and 2012 that evaluated trials in primary cardiovascular prevention and involved a randomized comparison of ASA versus placebo or control [13-15].

The first was carried out by Berger et al., who searched electronic databases (MEDLINE, the Cochrane Central Register of Controlled Trials, and EMBASE) for RCTs published up to 2011 [13]. The occurrence of a major cardiovascular event (MCE), i.e. non-fatal MIs, non-fatal strokes, all-cause and cardiovascular mortality, was chosen as primary outcome. As primary safety outcomes, the authors included major bleedings as defined by each study. All outcomes were analysed using the data reported in the original publications. Random effect meta-analysis was executed, using risk ratio (RR) as efficacy measure. Sensitivity analysis was performed and specific subsets of studies were analysed (removing one by one trials enrolling patients with diabetes or subclinical atherosclerosis and those including extended or controlled release aspirin). Meta-regression was applied to evaluate potential effect modifiers (year of study publication, baseline cardiovascular risk, mean age and sex of trial's participants, and dose of ASA). Potential publication biases were examined by constructing a funnel plot. Nine prospective randomized trials involving 102,621 participants $(52,145$ allocated to aspirin, 50,476 to placebo/control) were identified for inclusion and meta-analysed.

The same nine trials were incorporated in the metaanalysis by Raju et al. [14]. Medline, Cinahl, Embase and the Cochrane Library databases were sought up to May 2010, bibliographies of journal articles were hand-searched and experts were contacted to identify unpublished studies. Raju et al. considered the following outcomes 
(accepting primary trials investigators' definition): allcause mortality, cardiovascular mortality, major cardiovascular events (cardiovascular mortality, non-fatal MIs and non-fatal strokes), fatal or non-fatal MIs, fatal or nonfatal all-cause strokes, ischemic and haemorrhagic strokes, major and gastrointestinal bleedings. A risk of bias assessment using criteria adapted from the Cochrane Handbook on Systematic Reviews of Interventions [16] (proper generation of allocation sequence, proper concealment of the allocation sequence, blinding of participants, investigators, and outcome assessors, completeness of follow-up) was performed. Risk ratios (RR) were pooled with a random effect model and potential sources of statistical heterogeneity were explored, considering effect sizes related to year of publication of the study, cardiovascular baseline risk, mean age and sex of participants, and dose of ASA. Sensitivity analysis was performed excluding studies of lower quality (open label or with an incomplete follow-up), trials using higher doses of ASA (>150 mg/day), and those completed before 2000 (when different co-therapies for cardiovascular risk factors were supposed to be used). A total of 100,076 patients (50,868 in aspirin arm and 49,208 in placebo/control group) were included, because warfarin arms from factorial TPT study were excluded.

Finally, Seshasai et al. [15] searched PubMed and Cochrane Library electronic databases up to June 2011 for primary prevention randomized trials that included at least 1,000 participants, had at least 1-year of follow-up, recorded as main end-point CVDs and provided details of bleeding events. Primary efficacy end-point considered in the meta-analysis was total coronary heart diseases (CHD), whereas subtypes of vascular diseases, total CVD events, cause-specific deaths and all-cause mortality were secondary outcomes. As composite primary safety end point clinically "nontrivial" bleeding events were chosen, including fatal bleedings from any site, cerebrovascular or retinal bleedings from hollow viscus, bleedings requiring hospitalization or transfusion or study-defined major bleedings regardless of source. Seshasai et al. also analysed the effect of ASA in cancer prevention, but we decided not to report data, as we commented on the topic in a previous article [17]. The quality of the studies was evaluated using a Delphi scoring system based on the adequacy of randomization, allocation concealment, balance between groups at baseline, a priori identification of inclusion criteria, presence or absence of blinding, use of intent-to-treat analyses and reporting of point estimates and measures of variability. Potential publication bias was investigated with the use of funnel plots and by the Egger test. Combined odds ratios (ORs) were obtained by a random effects metaanalysis. Subgroup analyses considering participants' characteristics (mean age, percentage of men, smokers and diabetic participants, mean cholesterol levels and systolic blood pressure, dose of ASA administered) and sensitivity analysis excluding studies published before 2000, performed in non-Western population or exclusively in diabetics, people with peripheral arterial disease or health professionals were carried on. Seshasai et al. retrieved the nine randomized prevention trials analysed in the two previous meta-analyses. Like in Raju et al. [14], 102,621 patients (52,145 and 50,476 in aspirin and control group, respectively) were included.

Overall, the population studied was clinically heterogeneous. Original trials considered different cardiovascular risk profiles and administered different ASA doses (from $100 \mathrm{mg}$ every other day to $500 \mathrm{mg}$ daily). Three of the nine studies did not enroll women [18-20], whereas another one exclusively comprised them [12]. Two trials recruited only patients with diabetes mellitus [10, 12], one enrolled patients with a negative anamnesis for symptomatic cardiovascular diseases but with an ankle-brachial index $<0.95$ [11], and, finally, two studies included a small proportion of participants $(<5 \%)$ with a history of ischemic heart disease, stroke or transient ischemic attack [18, 19-21].

After carefully reading the description of the outcomes, we decided to classify similar end-points from single metaanalyses with a same label. The results of the three systematic reviews are reported in Table 1; they look roughly alike. The little gaps among the data are due to different populations and follow ups considered. Overall, ASA in primary prevention of cardiovascular diseases decreases the risk of total CVD events, but it mainly increases the possibility of major bleedings and is not associated with a significant reduction in all-cause or cardiovascular mortality (Table 1). No substantial results' modification was retrieved with subgroup analysis or meta-regression. Sensitivity analysis showed that there was no difference when (1) subjects with diabetes, asymptomatic PAD, or healthcare professionals or (2) studies considering a dose of aspirin major than $150 \mathrm{mg}$ day or (3) studies conducted before 2000 were excluded. Finally, the results are similar using fixed- or random-effect model.

Seshasai et al. [15] evaluated the absolute benefit versus adverse effects risk of ASA treatment according to the absolute event rates in the placebo arms. Both the net benefit related to the reduction of CVD events risk and the frequence of nontrivial bleeding events increased as absolute CVD event rates at baseline raised, but the net CVD benefit exceeded the bleeding risk at higher baseline CVD event rate. Statistical heterogeneity (quantified with Higgins test [22] and expressed as $I^{2}$ ) was relevant only for few outcomes (66\% for myocardial infarction and $37 \%$ for gastrointestinal bleeding) [13, 14]. 


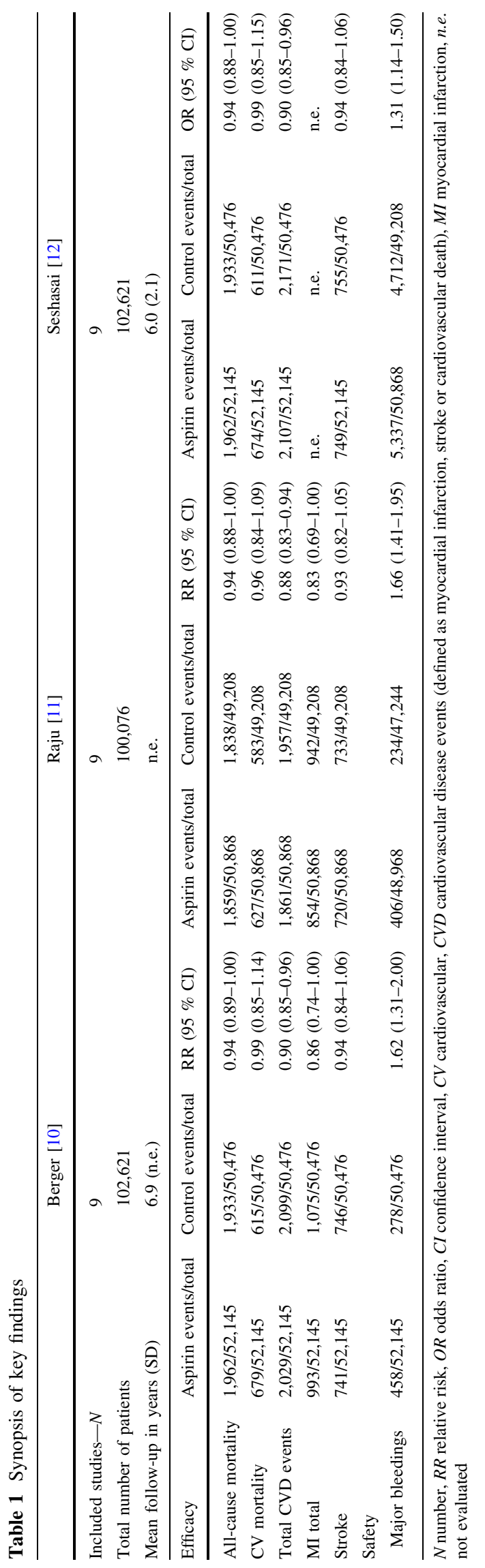

In summary, Berger et al. [13] commented on their work stating that the decision to use aspirin for MCE primary prevention remains a complex issue, requiring careful consideration. They reported for total CVD events an NNT of 253 and for major bleedings an NNH of 261 with a mean follow-up period of 6.9 years. Raju et al. [14] described the results as relative risk reduction (RRR) without considering the statistical significance of the data or their CIs, and declared that long-term treatment with ASA reduces allcause mortality (RRR $6 \%$ ) and MI (RRR $17 \%$ ). Finally, Seshasai et al. [15] reported an NNT for total CVD events of 120 and an NNH for major bleedings of 73 with a mean follow-up period of 6.0 years. They concluded that, because the benefit of aspirin treatment is accompanied by a significant increase in the possibility of bleeding, further studies are needed to identify subsets of participants having favourable risk to benefit ratio or involving patients at higher risk.

\section{Strengths of the studies}

- The three meta-analyses address a debated and clinically relevant topic; it is well known that cardiovascular diseases are the first cause of death in developed world.

- Evaluation of benefit versus risk is performed.

- Sources of heterogeneity are assessed and sensitivity analysis is carried on.

- Raju et al. [14] and Seshasai et al. [15] report a clear search strategy and study selection process.

\section{Weaknesses of the studies}

- We are aware that sensitivity analysis show the robustness of results removing trials at higher risk of bias, but comparisons in the studies included in metaanalyses could be questionable. Seshasai et al. [15] and Berger et al. [13] analyze all data from TPT trial [18], including also those from the aspirin or placebo associated with warfarin arms. In that study anticoagulation level obtained with warfarin is low (mean INR around 1.5) but the group treated with warfarin shows a risk of major gastrointestinal bleedings significantly higher than placebo arm.

- In the work by Berger et al. [13] the search strategy to identify studies is not shown: so they performed a metaanalysis without describing in detail the systematic review process.

- Raju et al. [14] report relative risk reduction marking it as significant, but they don't calculate any statistical difference test or confidence intervals nor did they relay subgroup results. 


\section{Question marks}

- A trend towards a reduction in all-cause mortality is observed: although the effect is modest and statistically non-significant $(-6 \%, 95 \%$ IC $0.88-1.00)$, considering the importance of the outcome and virtual benefit of ASA further investigations are mandatory to clarify this pivotal issue.

- The population enrolled in primary RCTs are heterogeneous, as it spans from healthy male doctors to patients with subclinical atherosclerosis, and finally to general population, and also CVD baseline risks are different. Relative risk or odds ratio permit to compare different risk population, but in this situation NNT should be calculated according to the different baseline risks. Thus, it may be helpul to use individual data to evaluate NNT and NNH in different subgroups (e.g. stratifying by CVD risk, age, co-morbidity) to identify the groups where the occurrence of adverse events is minimal.

- Three of the nine studies analysed included patients with low ankle brachial index. The detection of subclinical vascular lesion in asymptomatic people is a predictive factor of cardiovascular disease [23]. Even if the exclusion of these studies does not affect the results (as declared by the authors), we wonder whether they should be included in the meta-analyses.

\section{Sponsorships}

- Berger's research was supported by Astra Zeneca [13]. Raju et al. [14] and Seshasai et al. [15] did not report funding for the study.

\section{Clinical bottom line}

- The available evidence doesn't support the routine use of ASA for primary prevention of CVD in general population. The benefits in reducing CVD are almost perfectly equivalent to the adverse effect. The riskbenefit ratio should be weighed in every subject. In fact, it is reasonable to speculate that patients at moderatehigh risk for CVD (i.e. above $1 \%$ CVD event rate per year) and low bleeding risk can benefit from the use of ASA. This therapeutic strategy should be confirmed by further investigations.

\section{Conflict of interest None.}

\section{References}

1. Anderson J, Adams C, Antman E (2007) ACC/AHA 2007 guidelines for the management of patients with unstable angina/ non ST-elevation myocardial infarction: a report of the American College of Cardiology/American Heart Association Task Force on Practice Guidelines (Writing Committee to Revise the 2002 Guidelines for the management of patients with unstable angina/ non ST-elevation myocardial infarction): developed in collaboration with the American College of Emergency Physicians, the Society for Cardiovascular Angiography and Interventions, and the Society of Thoracic Surgeons: endorsed by the American Association of Cardiovascular and Pulmonary Rehabilitation and the Society for Academic Emergency Medicine. Circulation 116(7):e148-e304

2. Antman E, Hand M, Armstrong P (2008) 2007 focused update of the ACC/AHA 2004 guidelines for the management of patients with ST-elevation myocardial infarction: a report of the American College of Cardiology/American Heart Association Task Force on Practice Guidelines. Circulation 17(2):296-329

3. Patrono C, Baigent C, Hirsh J, Roth G, American College of Chest Physicians (2008) Antiplatelet drugs: American College of Chest Physicians Evidence-Based Clinical Practice Guidelines (8th Edition). Chest 133(6S):199S-233S

4. Smith S, Allen J, Blair S et al (2006) AHA/ACC guidelines for secondary prevention for patients with coronary and other atherosclerotic vascular disease: 2006 update: endorsed by the National Heart, Lung, and Blood Institute. Circulation 113(19): 2363-2372

5. Bartolucci A, Howard G (2006) Meta-analysis of data from the six primary prevention trials of cardiovascular events using aspirin. Am J Cardiol 98(6):746-750s

6. Berger J, Roncaglioni M, Avanzini F, Pangrazzi I, Tognoni G, Brown D (2006) Aspirin for the primary prevention of cardiovascular events in women and men: a sex-specific meta-analysis of randomized controlled trials. JAMA 295(3):306-313

7. Antithrombotic Trialists' (ATT) Collaboration, Baigent C, Blackwell L, Collins R et al (2009) Aspirin in the primary and secondary prevention of vascular disease: collaborative metaanalysis of individual participant data from randomised trials. Lancet 373(9678): 1849-1860

8. US Preventive Services Task Force (2009) Aspirin for the prevention of cardiovascular disease: US Preventive Services Task Force recommendation statement. Ann Intern Med 150(6):396404

9. Pearson T, Blair S, Daniels S et al (2002) AHA guidelines for primary prevention of cardiovascular disease and stroke: 2002 update: consensus panel guide to comprehensive risk reduction for adult patients without coronary or other atherosclerotic vascular diseases. American Heart Association Science Advisory and Coordinating Committee. Circulation 106(3):388-391

10. Belch J, MacCuish A, Campbell I et al. (2008) The prevention of progression of arterial disease and diabetes (POPADAD) trial: factorial randomised placebo controlled trial of aspirin and antioxidants in patients with diabetes and asymptomatic peripheral arterial disease. BMJ 337 (a1840)

11. Fowkes F, Price J, Stewart M et al (2010) Aspirin for prevention of cardiovascular events in a general population screened for a low ankle brachial index: a randomized controlled trial. JAMA 303(9):841-848

12. Ogawa H, Nakayama M, Morimoto $T$ et al (2008) Low-dose aspirin for primary prevention of atherosclerotic events in patients with type 2 diabetes: a randomized controlled trial. JAMA 300(18):2134-2141 
13. Berger J, Lala A, Krantz M, Baker G, Hiatt W (2011) Aspirin for the prevention of cardiovascular events in patients without clinical cardiovascular disease: a meta-analysis of randomized trials Am Heart J 162(1):115.e112-124.e112

14. Raju N, Sobieraj-Teague M, Hirsh J, O’Donnell M, Eikelboom J (2011) Effect of aspirin on mortality in the primary prevention of cardiovascular disease. Am J Med 124(7):621-629

15. Seshasai S, Wijesuriya S, Sivakumaran R et al (2012) Effect of aspirin on vascular and nonvascular outcomes: meta-analysis of randomized controlled trials. Arch Intern Med 172(3):209_ 216

16. Higgins J, Altman D, Sterne J (2011) Chapter 8: assessing risk of bias in included studies. In: Higgins JPT Green S (eds) Cochrane handbook for systematic reviews of interventions version 5.1.0. The Cochrane Collaboration

17. Tagliabue L, Pugliano M (2011) Will aspirin rescue us from cancer? Intern Emerg Med 6(5):449-451

18. AAVV (1998) Thrombosis prevention trial: randomised trial of low-intensity oral anticoagulation with warfarin and low-dose aspirin in the primary prevention of ischaemic heart disease in men at increased risk. The Medical Research Council's General Practice Research Framework. Lancet 351(9098):233-241
19. AAVV (1989) Final report on the aspirin component of the ongoing physicians' health study. Steering Committee of the Physicians' Health Study Research Group. N Engl J Med 321(3):129-135

20. Peto R, Gray R, Collins R et al (1988) Randomised trial of prophylactic daily aspirin in British male doctors. BMJ 296(6618):313-316w

21. Hansson L, Zanchetti A, Carruthers S et al (1998) Effects of intensive blood-pressure lowering and low-dose aspirin in patients with hypertension: principal results of the hypertension optimal treatment (HOT) randomised trial. HOT Study Group. Lancet 351(9118):1755-1762

22. Higgins J, Thompson S, Deeks J, Altman D (2003) Measuring inconsistency in meta-analyses. BMJ 327:557-560

23. Stein JH, Korcarz CE, Hurst RT, Lonn E, Kendall CB, Mohler ER, Najjar SS, Rembold CM, Post WS (2008) Use of carotid ultrasound to identify subclinical vascular disease and evaluate cardiovascular disease risk: a consensus statement from the American Society of echocardiography carotid intima-media thickness task force. Endorsed by the Society for Vascular Medicine. J Am Soc Echocardiogr 21(2):93-111 (quiz 189-190). doi:10.1016/j.echo.2007.11.011 